\title{
Severe Fever with Thrombocytopenia Syndrome Virus Infection, South Korea, 2010
}

\section{Young Ree Kim, ${ }^{1}$ Yeojun Yun, ${ }^{1}$ Seung Geon Bae, Dahee Park, Suhyun Kim, Jae Myun Lee, Nam-Hyuk Cho, Yang Soo Kim, Keun Hwa Lee}

Author affiliations: Jeju National University College of Medicine, Jeju, South Korea (Y.R. Kim, S.G. Bae, D. Park, S. Kim, K.H. Lee); Ewha Womans University, Seoul, South Korea (Y. Yun); Yonsei University College of Medicine, Seoul (J.M. Lee); Seoul National University College of Medicine, Seoul (N.-H. Cho); University of Ulsan College of Medicine, Seoul (Y.S. Kim).

DOI: https://doi.org/10.3201/eid2411.170756

Severe fever with thrombocytopenia syndrome (SFTS) was reported in China in 2009 and in South Korea in 2012. We found retrospective evidence of SFTS virus infection in South Korea in 2010, suggesting that infections in South Korea occurred before previously reported and were more concurrent with those in China.

Cevere fever with thrombocytopenia syndrome virus (SFTSV) is a tickborne virus (genus Phlebovirus, family Phenuiviridae) that can cause hemorrhagic fever $(1,2)$. Severe fever with thrombocytopenia syndrome (SFTS) was confirmed in China in 2009 (2) and then retrospectively reported in South Korea in 2012 and in western Japan in $2013(3,4)$. Most SFTSV infections occur through bites from Haemaphysalis longicornis ticks, although transmission can also occur through close contact with an infected patient (5-7). We provide retrospective evidence of SFTSV infections in South Korea from 2010, confirming that infections in South Korea occurred earlier than previously reported and were more concurrent with the first reported infections in China.

For this study, we used stored serum samples from 58 patients who had high erythrocyte sedimentation rates (ESRs) and were admitted to Jeju National University Hospital in Jeju, South Korea, during July 2010; SFTS was not a criterion for selection. Jeju is a high-prevalence region for SFTS, and July is the month with the highest SFTS prevalence in South Korea. The major clinical signs and symptoms of SFTS are an acute and high fever, thrombocytopenia, leukopenia, elevated serum hepatic enzyme levels, gastrointestinal symptoms, and multiorgan failure, with a death rate of $12 \%-30 \%$. However, atypical signs

${ }^{1}$ These authors contributed equally to this article. and symptoms of SFTS have been identified, and asymptomatic infections among humans have been reported in South Korea $(3,6,8)$.

For molecular diagnosis of SFTSV, we extracted RNA from serum by using the QIAamp Viral RNA Mini Kit (QIAGEN, Hilden, Germany). We performed real-time reverse transcription PCR (RT-PCR) to amplify the partial small (S) segment of the viral RNA from the stored serum and confirm SFTSV infection (9). We sequenced real-time RT-PCR products using the BigDye Terminator Cycle Sequencing kit (Perkin Elmer Applied Biosystems, Warrington, UK). We conducted phylogenetic analysis of partial S segment sequences using MEGA6 (10) and constructed phylogenetic trees using the maximum-likelihood method (Figure).

Our results showed 2 positive results from the stored serum of 2 patients. Neither patient reported history of travel to other endemic countries, such as China or Japan.

Patient Jeju 01-South Korea-07-2010 (no. 430), a 77 -year-old man, had diabetes and hypertension for 45 years. The patient had received a diagnosis of end-stage renal disease 7 years before admission and was on hemodialysis; he experienced an episode of gout 5 years before admission. The patient came to the hospital's orthopedic outpatient clinic reporting that he had pain, swelling, and febrile sensation of the right lateral malleolus area for several days. He had undergone orthopedic surgery with debridement for a wound of the right lateral malleolus area caused by an electric plate burn 1.5 years earlier. He was retired, lived in Jeju, and was admitted to the hospital for emergency orthopedic surgery. Physical examination recorded body temperature of $37.2^{\circ} \mathrm{C}$, blood pressure 140/60 $\mathrm{mm} \mathrm{Hg}$, heart rate 78/min, and respiratory rate 20 breaths/min. Initial laboratory data revealed slight thrombocytopenia $\left(199,000\right.$ platelets $\left./ \mathrm{mm}^{3}\right)$, and absolute neutrophil count (ANC) was 7,374. Alkaline phosphatase (ALP), aspartate aminotransferase (AST), and alanine aminotransferase (ALT) levels were within reference ranges; however, ESR $(112 \mathrm{~mm} / \mathrm{h})$, blood urea nitrogen $(24.8 \mathrm{mg} /$ $\mathrm{dL})$, and creatinine $(4.8 \mathrm{mg} / \mathrm{dL})$ were elevated.

Patient Jeju 02-South Korea-07-2010 (no. 468), a 76-year-old woman, visited the hospital's orthopedic outpatient clinic reporting pain in both knees for 10 years. She was a housewife, lived in Jeju, and had received temporary symptom treatment from local clinics. She was admitted to the hospital for total knee replacement arthroplasty. Her only underlying disease was diabetes. Physical examination recorded body temperature of $36.8^{\circ} \mathrm{C}$, blood pressure $120 / 60 \mathrm{~mm} \mathrm{Hg}$, heart rate $68 / \mathrm{min}$, and respiratory rate 20 breaths/minute. Initial laboratory test results were within reference ranges except for urine glucose $(4+)$; platelet count was $620,000 / \mathrm{mm}^{3}$, ANC was 2,065 , and ALP, AST, and ALT levels were within reference ranges. Her ESR level was elevated, at $74 \mathrm{~mm} / \mathrm{h}$. 


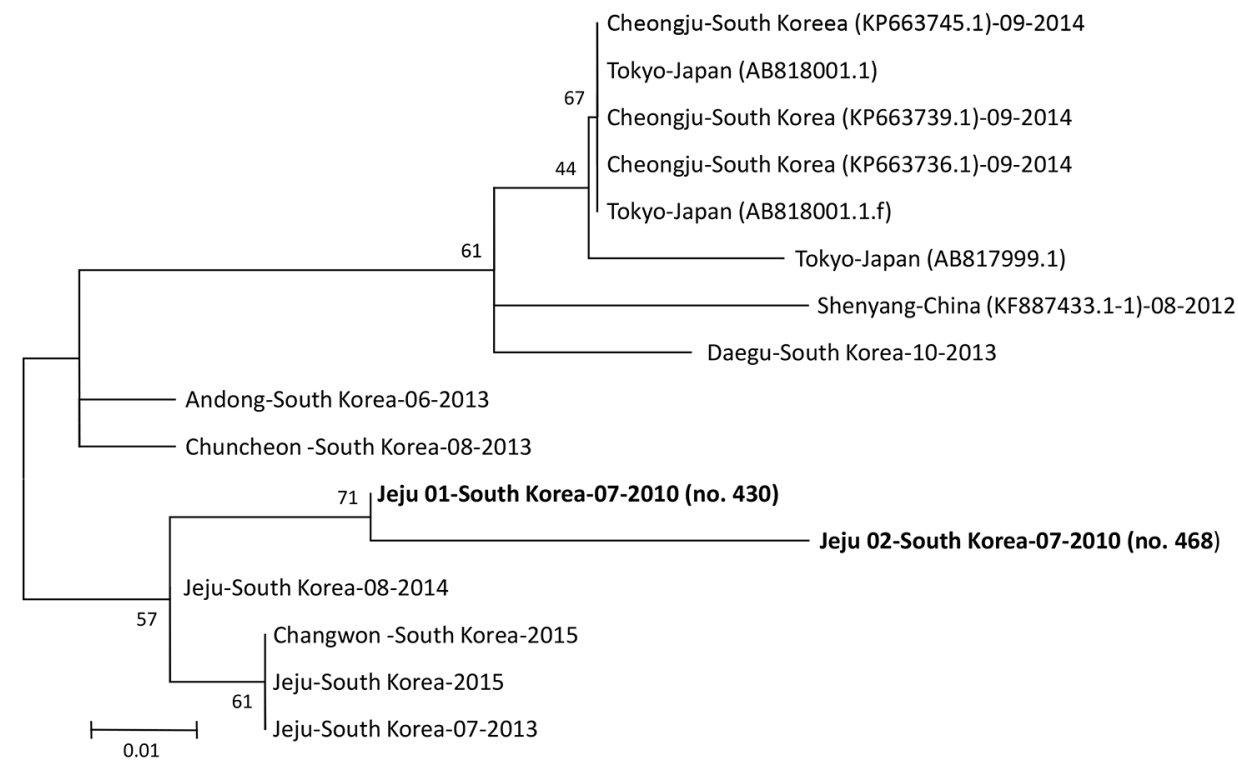

Figure. Phylogenetic tree constructed based on partial small segment sequences of severe fever with thrombocytopenia syndrome virus identified in stored serum samples collected in 2010 from 2 patients in South Korea (bold) compared with reference viruses. We constructed the tree using the maximum-likelihood method with MEGA 6 (10). The partial small sequence data for the viruses identified in China, South Korea, and Japan were obtained from GenBank (accession numbers in parentheses). Scale bar indicates nucleotide substitutions per site.
SFTSV was reported in South Korea in 2012 (3). In this study, we retrospectively confirmed 2 SFTSV infections in South Korea in 2010 by amplification of the partial $\mathrm{S}$ segment of the viral RNA from stored serum of patients with a high ESR. Atypical signs and symptoms of SFTS among patients and asymptomatic infections have previously been reported in South Korea $(3,6,8)$. In this study, we found that the signs and symptoms of SFTS were distinct from the major clinical signs and symptoms of SFTS, and the knee pain was coincidence. However, a case study showed that 1 SFTS patient had a crusted erythematous ulcer on the lateral side of the left knee, accompanied with left inguinal lymphadenopathy.

In conclusion, we suggest that SFTSV infections in South Korea have occurred during a period similar to the period reported for China, where SFTSV was found in 2009. In addition, the signs and symptoms of SFTS may be atypical. Therefore, further clinical, epidemiologic, and laboratory research is needed to better understand the transmission dynamics of SFTSV and prevent additional SFTSV infections in other populations.

\section{Acknowledgments}

We thank L. Bakkensen for providing comments on this article.

This work was supported by a grant from the Korean Health Technology R\&D Project of the Ministry of Health and Welfare, South Korea (grant no. HI15C2891).

\section{About the Authors}

Dr. Kim is a professor at the Department of Laboratory Medicine, Jeju National University College of Medicine, Jeju, South Korea. Dr. Yun is a research professor at Ewha
Medical Research Institute, Ewha Womans University, Seoul, South Korea. Their primary research interest is clinical microbiology.

\section{References}

1. International Committee on Taxonomy of Viruses (ICTV). Taxonomy. 2017 [cited 2017 May 3]. https://talk.ictvonline.org/ taxonomy

2. Yu XJ, Liang MF, Zhang SY, Liu Y, Li JD, Sun YL, et al. Fever with thrombocytopenia associated with a novel bunyavirus in China. N Engl J Med. 2011;364:1523-32. http://dx.doi.org/ 10.1056/NEJMoa1010095

3. Kim KH, Yi J, Kim G, Choi SJ, Jun KI, Kim NH, et al. Severe fever with thrombocytopenia syndrome, South Korea, 2012. Emerg Infect Dis. 2013;19:1892-4. http://dx.doi.org/10.3201/ eid1911.130792

4. Takahashi T, Maeda K, Suzuki T, Ishido A, Shigeoka T, Tominaga $\mathrm{T}$, et al. The first identification and retrospective study of severe fever with thrombocytopenia syndrome in Japan. J Infect Dis. 2014;209:816-27. http://dx.doi.org/10.1093/infdis/ jit603

5. Liu Q, He B, Huang SY, Wei F, Zhu XQ. Severe fever with thrombocytopenia syndrome, an emerging tick-borne zoonosis. Lancet Infect Dis. 2014;14:763-72. http://dx.doi.org/10.1016/ S1473-3099(14)70718-2

6. Yoo JR, Heo ST, Park D, Kim H, Fukuma A, Fukushi S, et al. Family cluster analysis of severe fever with thrombocytopenia syndrome virus infection in Korea. Am J Trop Med Hyg. 2016;95:1351-7. http://dx.doi.org/10.4269/ajtmh.16-0527

7. Bao CJ, Guo XL, Qi X, Hu JL, Zhou MH, Varma JK, et al. A family cluster of infections by a newly recognized bunyavirus in eastern China, 2007: further evidence of person-to-person transmission. Clin Infect Dis. 2011;53:1208-14. http://dx.doi.org/ $10.1093 / \mathrm{cid} / \mathrm{cir} 732$

8. Wi YM, Woo HI, Park D, Lee KH, Kang C-I, Chung DR, et al Severe fever with thrombocytopenia syndrome in patients suspected of having scrub typhus. Emerg Infect Dis. 2016;22: 1992-5. http://dx.doi.org/10.3201/eid2211.160597

9. Zhang YZ, He YW, Dai YA, Xiong Y, Zheng H, Zhou DJ, et al. Hemorrhagic fever caused by a novel bunyavirus in China: 
pathogenesis and correlates of fatal outcome. Clin Infect Dis. 2012;54:527-33. http://dx.doi.org/10.1093/cid/cir804

10. Tamura K, Stecher G, Peterson D, Filipski A, Kumar S. MEGA6: Molecular Evolutionary Genetics Analysis version 6.0. Mol Biol Evol. 2013;30:2725-9. http://dx.doi.org/10.1093/molbev/ mst197

Address for correspondence: Keun Hwa Lee, Department of Microbiology and Immunology, Jeju National University College of Medicine, 15, Aran 13-gil, Jeju 63241, South Korea; email: yomust7@jejunu.ac.kr

\section{Spotted Fever Group Rickettsiae in Inner Mongolia, China, 2015-2016}

\author{
Gaowa, Wulantuya, Xuhong Yin, Shengchun Guo, \\ Chunlian Ding, Minzhi Cao, Hiroki Kawabata, \\ Kozue Sato, Shuji Ando, Hiromi Fujita, \\ Fumihiko Kawamori, Hongru Su, \\ Masahiko Shimada, Yuko Shimamura, \\ Shuichi Masuda, Norio Ohashi
}

\begin{abstract}
Author affiliations: College of Hetao, Bayan Nur City, Inner Mongolia, China (Gaowa, Wulantuya, X. Yin, S. Guo, C. Ding); Bayan Nur Centers for Disease Control and Prevention, Bayan Nur City (M. Cao); National Institute of Infectious Diseases, Shinjuku-ku, Tokyo, Japan (H. Kawabata, K. Sato, S. Ando); Mahara Institute of Medical Acarology, Anan City, Tokushima, Japan (H. Fujita); University of Shizuoka, Shizuoka City, Japan (F. Kawamori, H. Su, M. Shimada, Y. Shimamura, S. Masuda, N. Ohashi)
\end{abstract}

DOI: https://doi.org/10.3201/eid2411.162094

We found Rickettsia raoultii infection in 6/261 brucellosisnegative patients with fever of unknown origin in brucellosis-endemic Inner Mongolia, China. We further identified Hyalomma asiaticum ticks associated with $R$. raoultii, $H$. marginatum ticks associated with $R$. aeschlimannii, and Dermacentor nuttalli ticks associated with both rickettsiae species in the autonomous region.

Qpotted fever group rickettsiae (SFGR) are vector$\checkmark$ borne pathogens. In China, 5 SFGR genotypes have been identified as causative agents of human rickettsiosis: $R$. heilongjiangensis, $R$. sibirica subsp. sibirica BJ-90,
Candidatus Rickettsia tarasevichiae, $R$. raoultii, and Rickettsia sp. XY99 (1-4).

Brucellosis, a zoonotic disease, is highly endemic to Inner Mongolia, China, and is increasing in workers in agriculture or animal husbandry (5). However, some agriculture workers with brucellosis-like symptoms, including general malaise and fever, were seronegative for Brucella spp. We suspected that fever of unknown origin among brucellosis-seronegative patients might be caused by tickborne pathogens. We identified 6 cases of human $R$. raoultii infections in brucellosis-seronegative patients in western Inner Mongolia, and we investigated exposure to ticks infected with SFGR.

During 2015-2016, we obtained 261 blood samples from brucellosis-seronegative patients with fever of unknown origin in Bayan Nur Centers for Disease Control and Prevention (Bayan Nur City, Inner Mongolia, China). The review board of the Department of Medicine at College of Hetao (Bayan Nur City) approved the study. We extracted DNA from each blood sample using the DNeasy Mini Kit (QIAGEN, Hilden, Germany) and conducted PCR targeting SFGR gltA (6). The PCR primers used, gltA-Fc (5'-CGAACTTACCGCTATTAGAATG-3') and gltA-Rc (5'-CTTTAAGAGCGATAGCTTCAAG-3'), were described previously (4). We designed the primers $16 \mathrm{~S}$ rDNA R-2F (5'-GAAGATTCTCTTTCGGTTTCGC-3'), 16S rDNA R-2R (5'-GTCTTGCTTCCCTCTGTAAAC-3'), rompA-Fb (5'-GGTGCGAATATAGACCCTGA-3'), and rompA-Ra (5'-TTAGCTTCAGAGCCTGACCA-3') for this study and deposited the sequences obtained of glt $A$, ompA, and $16 \mathrm{~S}$ rDNA into GenBank (accession nos. MH267733-47). We used genomic DNA extracted from L929 cells infected with Rickettsia sp. LON-13 (gltA: AB516964) as a positive control.

We detected gltA amplicons from 6/261 (2.3\%) blood samples (Table). All 6 patients had strong malaise and mild fever of $36.8^{\circ} \mathrm{C}-37.3^{\circ} \mathrm{C}$ but no rash. Five of these patients also had arthralgia and vomiting.

Sequence and phylogenetic analysis showed that the sequences of 6 nearly full-length $(1.1 \mathrm{~kb}) \mathrm{glt} A$ amplicons with were identical to each other and to $R$. raoultii gltA (GenBank accession no. DQ365803). We further analyzed ompA and $16 \mathrm{~S}$ rDNA in gltA-positive samples. All 6 samples were PCR positive for both genes; 552-bp sequences of the amplicons were identical to sequences of $R$. raoultii ompA (GenBank accession no. AH015610), and 389-bp sequences of the amplicons were identical to sequences of $R$. raoultii $16 \mathrm{~S}$ rDNA (GenBank accession no. EU036982). PCR results were negative for the genes Anaplasma phagocytophilum p44/msp2, Ehrlichia chaffeensis p28/omp-1, and Borrelia spp. flaB. An indirect immunofluorescence assay showed that $\operatorname{IgM}$ and IgG titers against $R$. japonica were $40-80$ for IgM in 3 patients and 160 for $\mathrm{IgG}$ in 2 patients. 